\title{
Tension/compression test of auto-body steel sheets with the variation of the pre-strain and the strain rate
}

\author{
G. H. Bae \& H. Huh \\ School of Mechanical, Aerospace and Systems Engineering, KAIST, \\ Republic of Korea
}

\begin{abstract}
This paper investigates the tension/compression hardening behaviour of autobody steel sheets with the variation of the pre-strain and the strain rate. To conduct tension/compression tests with the variation of the pre-strain and the strain rate, an experimental method was established by using a newly developed clamping device to suppress buckling of a specimen. The clamping device provides the supporting force from compression-type coil springs during the test with a conventional dynamic material fatigue testing machine. From experiments, the tension/compression hardening behaviour was observed with the variation of the pre-strain and the strain rate. Effects of the pre-strain and the strain rate on the hardening behaviour were also investigated based on the tension/compression test results.
\end{abstract}

Keywords: tension/compression test, clamping device, pre-strain, strain rate.

\section{Introduction}

Spring-back caused by the elastic recovery of the residual stress inside a formed part has been one of the most significant sources of defects in the sheet metal forming process in recent years. Spring-back predictability of numerical simulation, however, has not been satisfactory because its performance is not good enough to calculate accurate residual stress during the forming process. To improve spring-back predictability in numerical simulation, many researchers have been trying to use accurate information of the hardening behaviour of sheet materials in finite element analysis. The hardening behaviour of sheet materials 
has been measured with various mechanical testing methods in the quasi-static region [1-3]. It has been successful to predict spring-back accurately with numerical simulation by applying the precise hardening behaviour obtained from tension/compression tests at the quasi-static state $[4,5]$.

Numerical simulation still cannot accurately predict the final shape of the formed part after spring-back for a higher strain rate because of lack in information about the tension/compression hardening behaviour at high strain rates. To enhance spring-back predictability in numerical simulation for actual formed parts, the accurate hardening behaviour should be applied in finite element analysis by performing tension/compression tests with the variation of the strain rate as well as of the pre-strain.

This paper investigates the tension/compression hardening behaviour of autobody steel sheets with the variation of the pre-strain and the strain rate. To conduct tension/compression tests, a simple clamping device was newly developed to suppress buckling of a specimen during the compression loading. The clamping device provides the side force from compression-type coil springs. The compression-type coil springs were selected for the desired clamping pressure calculated by the plate buckling theory adopted by Cao and Wang [6]. Based on the secant formula and the Euler method adopted by Boger et al. [2], specimen dimensions were also designed to prevent buckling along the width direction in the gauge section and along the longitudinal direction in the unclamped region. The strain in the gauge region of the specimen was measured directly by the digital image processing technique. The hardening behaviour of auto-body steel sheets was investigated precisely based on the tension/compression test results with the variation of the pre-strain and the strain rate.

\section{Test preparation}

To obtain a larger compressive strain range in the tension/compression test, buckling of a specimen should be prevented by using a properly-designed specimen and by imposing a sufficient clamping force. Fig. 1 shows three representative buckling modes in the tension/compression test: (1) buckling in the thickness direction in the gauge region (T-buckling); (2) buckling in the

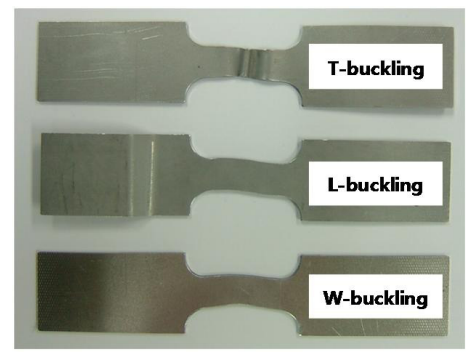

Figure 1: Three representative buckling modes in the tension/compression test. 
unclamped region (L-buckling); (3) buckling in the width direction in the gauge region (W-buckling). T-buckling mode can be suppressed by imposing a sufficient clamping force on a specimen with a special clamping device. Lbuckling and W-buckling modes can be prevented by designing an appropriate specimen shape.

\subsection{Specimen shape}

Boger et al. [2] used the secant formula and the Euler method to calculate the attainable compressive strain for L-buckling and W-buckling modes, respectively. When the flow curve is expressed by the Swift model, the attainable compressive strain for two buckling modes can be calculated as follows:

$$
\begin{aligned}
& \bar{\varepsilon}_{L}=\varepsilon_{0}\left[\left(\frac{B}{W}\right)^{\frac{1}{n}}-1\right] \\
& \bar{\varepsilon}_{W}=\frac{\pi^{2} n W^{2}}{3 G^{2}}-\varepsilon_{0}
\end{aligned}
$$

where $B$ and $W$ are the specimen width in the gripping region and the gauge region, respectively. $G$ is the gauge length of the specimen. $\varepsilon_{0}$ and $n$ are the plastic strain for the yield stress and the hardening exponent in the Swift model, respectively. If the gauge length of the specimen and the coefficients of the Swift model are given, the attainable compressive strains are a function of the gauge width. More detailed formulation procedure can be found in the reference [2].

The specimen design was performed using the above equations formulated for the attainable compressive strain of L-buckling and W-buckling. The selected steel sheets are SPCC and DP590 which are commonly used auto-body steel sheets. Strains at uniform elongation before necking initiates are approximately 0.15 and 0.12 for SPCC and DP590, respectively. These values are assigned as the maximum pre-strain for tension/compression tests without necking of a specimen. Table 1 shows the coefficients of the Swift model and r-values for SPCC and DP590 at a quasi-static state. A specimen shape for the uniaxial tensile test [7, 8] was utilized as reference specimen dimensions for tension/compression tests as shown in fig. 3(a). In order to reduce the required clamping force to suppress T-buckling, the gauge length is first reduced from 30 $\mathrm{mm}$ to $20 \mathrm{~mm}$. The small clamping force is favourable for the reliable tension/compression test, which can reduce the frictional and biaxial effect on a specimen. The attainable compressive strain was plotted with respect to the gauge width as shown in fig. 2. From the plotted curves, the optimal gauge width can be selected as $8.8 \mathrm{~mm}$ for SPCC and $8.2 \mathrm{~mm}$ for DP590, respectively. To ensure a stable testing region for the two steel sheets, the final gauge width can be determined to be $8.8 \mathrm{~mm}$ as shown in fig. 3(b). 
Table 1: $\quad$ Coefficients of the Swift model and r-values of SPCC and DP590.

\begin{tabular}{cccccccc}
\hline Material & $K[\mathrm{MPa}]$ & $\varepsilon_{0}$ & $n$ & $r_{0}$ & $r_{45}$ & $r_{90}$ & $R$ \\
\hline \hline SPCC & 555.772 & 0.01121 & 0.253 & 1.381 & 1.094 & 1.697 & 1.317 \\
DP590 & 1079.261 & 0.00879 & 0.220 & 0.704 & 0.784 & 0.948 & 0.805 \\
\hline
\end{tabular}

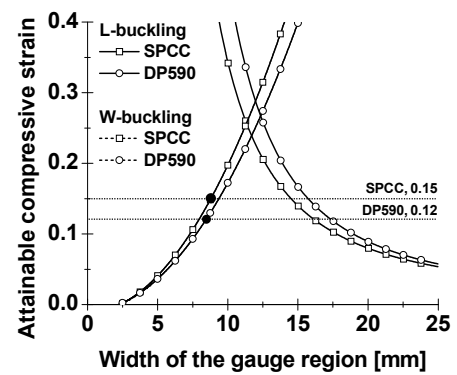

Figure 2: Attainable compressive strain with respect to the width at the gauge region for SPCC and DP590.

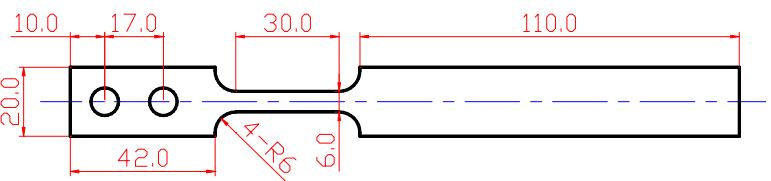

(a)

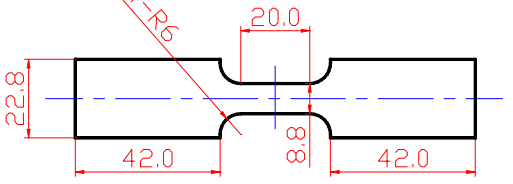

(b)

Figure 3: Specimen dimensions for the uniaxial tensile test and the tension/compression test: (a) uniaxial tension test [7, 8]; (b) tension/compression test (suggested).

\subsection{Clamping force}

Cao and Wang [6] proposed an equation to calculate the blank holding force to suppress flange wrinkling in the sheet metal forming process. To calculate the clamping force to suppress T-buckling of a specimen, the plate buckling theory was also employed by assuming the gauge region of a specimen to be a rectangular plate model. The critical normal force required to suppress buckling of a specimen can be calculated by 


$$
\begin{gathered}
F=\frac{3 K G W t}{4(n+1) \delta}\left[\begin{array}{l}
\left\{\varepsilon_{0}-\ln \left(1-\frac{2 u_{x}}{G}\right)\right\}^{n+1} \\
-\frac{2}{G}\left(\frac{c t}{2}+\varepsilon_{0}\left(\frac{1}{m^{2} \delta}+\frac{t}{2}\right)\right)^{n+1}\left(\frac{1}{m^{2} \delta}+\frac{t}{2}\right)^{-n} \tan ^{-1}(m \delta)
\end{array}\right] \\
\text { where } c=\frac{1+R}{\sqrt{1+2 R}}, m=\frac{2 \pi}{G-2 u_{x}}, \delta=\frac{\sqrt{2 u_{x}\left(l-u_{x}\right)}}{\pi}
\end{gathered}
$$

where $t$ and $u_{x}$ are the thickness of a specimen and a certain edge displacement. $K, n$ and $\varepsilon_{0}$ are coefficients of the Swift model. For the tension/compression test, the specimen thickness and width change in the gauge region during deformation. These values can be calculated based on anisotropy of material.

$$
\begin{gathered}
W=W_{0}-\frac{W_{0}}{1+R} \ln \left(1-\frac{2 u_{x}}{G}\right) \\
t=t_{0}-\frac{t_{0} R}{1+R} \ln \left(1-\frac{2 u_{x}}{G}\right)
\end{gathered}
$$

The critical normal force defined in eqn. (3) is now used to calculate the critical clamping force to suppress T-buckling in the tension/compression test. More detailed formulation procedure of the critical normal force can be found in the reference [6]. Fig. 4 shows the critical clamping force with respect to the compressive plastic strain. From the plotted curves, the required clamping force for SPCC and DP590 can be determined to be $2.003 \mathrm{kN}$ and $2.275 \mathrm{kN}$, respectively.

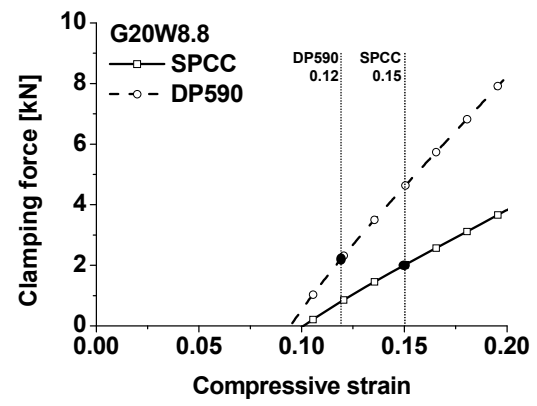

Figure 4: Required clamping force with respect to the compressive strain for SPCC and DP590.

\subsection{Design of a new clamping device}

Based on specimen dimensions and the required clamping force, a new clamping device was developed to suppress T-buckling of specimens. Fig. 5(a) gives a schematic diagram for the clamping device developed. A controllable clamping 
system was suggested by using compression-type coil springs. Four coil springs on each side compress a specimen by fastening the bolts up to the desired deflection distance which is determined by the gap controlling bar. Before the tension/compression test starts, the gap controlling bar is uninstalled from the clamping device in order to allow thickness change of the specimen during the tension/compression test. For lubrication between the specimen and the clamping plate, a Teflon film (3M 5490 model) with a thickness of $0.09 \mathrm{~mm}$ is attached on the clamping plate. A compression-type coil spring was selected based on the clamping force calculated in Section 2.2 for SPCC and DP590. The maximum clamping force was selected at $4 \mathrm{kN}$ to ensure performance of the device although the required clamping forces were $2.003 \mathrm{kN}$ for SPCC and $2.275 \mathrm{kN}$ for DP590.

To provide the required clamping forces, $\Phi 16 L 40$ coil spring was selected for the clamping device. The maximum load and the spring constant of the selected coil spring are $1.265 \mathrm{kN}$ and $0.158 \mathrm{kN} / \mathrm{mm}$, respectively.

The clamping device was manufactured with the specified dimensions based on the specimen shape and the spring size. As shown in fig. 5(b), the springloaded clamping device has a small and simple structure with light weight. The clamping force is controllable by determining the deflection distance of the coil springs. These advantages are favourable for easy and convenient tension/compression tests at high strain rates.

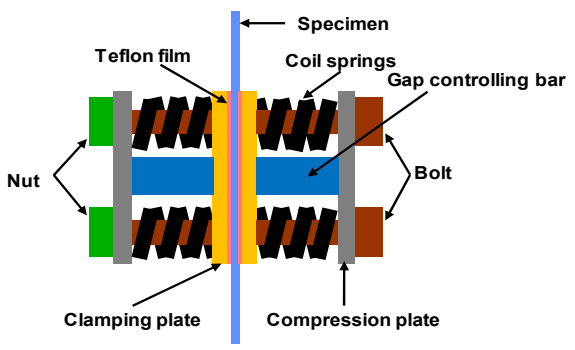

(a)

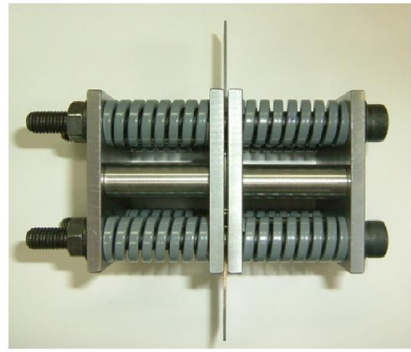

(b)

Figure 5: Spring-loaded clamping device for the tension/compression test: (a) schematic diagram; (b) manufactured clamping device.

\section{Experiments}

\subsection{Test material}

The tension/compression test of auto-body steel sheets was conducted with the variation of the pre-strain and the strain rate. Test materials were SPCC and DP590 with a thickness of $1.2 \mathrm{~mm}$. The chemical composition of the sheet metals is presented in table 2 .

Table 2: $\quad$ Chemical composition of SPCC and DP590 [wt.\%].

\begin{tabular}{cccccc}
\hline Material & $\mathrm{C}$ & $\mathrm{Mn}$ & $\mathrm{Si}$ & $\mathrm{P}$ & $\mathrm{S}$ \\
\hline \hline SPCC & 0.085 & 0.420 & 0.040 & 0.0012 & 0.0150 \\
DP590 & 0.326 & 1.700 & 0.123 & 0.0162 & 0.0009 \\
\hline
\end{tabular}




\subsection{Testing machine}

A clamped specimen is gripped by the dynamic material fatigue testing machine, INSTRON8801, to perform tension/compression tests with the variation of the pre-strain and the strain rate. The dynamic material fatigue testing machine shown in fig. 6 has a maximum stroke of $\pm 75 \mathrm{~mm}$ in the vertical direction actuated by a hydraulic system. The load cell installed in the testing machine can measure a load of up to $\pm 100 \mathrm{kN}$. The gripper is also actuated by a hydraulic system.

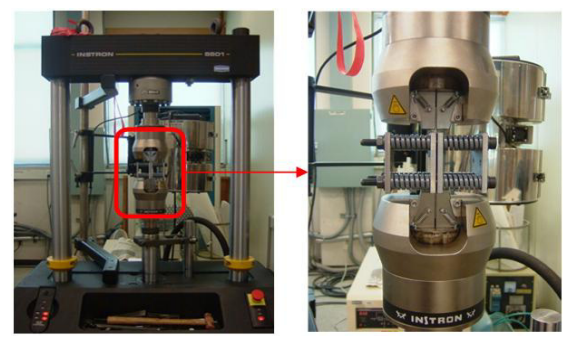

Figure 6: Tension/compression testing system utilizing the dynamic material fatigue testing machine, INSTRON8801, and the spring-loaded clamping device.

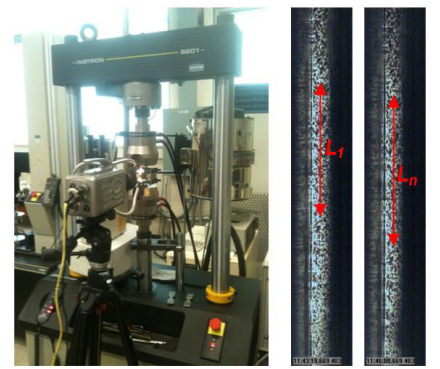

(a)

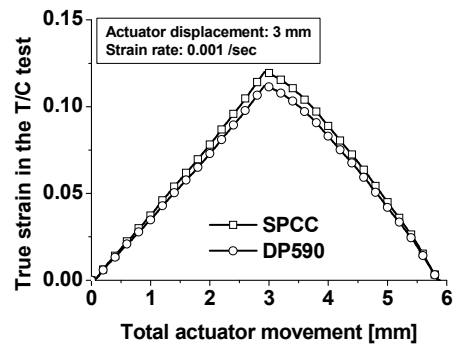

(b)

Figure 7: Strain measuring method using the digital image processing technique with the high speed camera: (a) recording of the deformation history; (b) measured strain during the tension/compression test.

\subsection{Strain measurement}

Because the specimen is fully covered by the clamping plates, conventional extensometers cannot be employed to measure the strain in the side of the gauge region during the tension/compression test. As an alternative, a digital image processing technique was utilized to measure the strain directly. Before the tension/compression test, a speckled pattern was sprayed on the side wall of the specimen as shown in fig. 7(a). A high speed camera captures a series of frames for deformation during the tension/compression test. The strain can be measured 
from a graph in fig. 7(b) obtained by analyzing recorded frames using the digital image processing technique.

\subsection{Tension/compression test}

Stable tension/compression testing conditions were established by comparing the response of the testing machine with the variation of the actuator displacement and the strain rate. Table 3 presents experimental conditions for the tension /compression test. From the experiments, load-displacement curves and strains in the gauge region were determined for various pre-strains and strain rates. The tension/compression hardening curves can be obtained after correction procedures to subtract the frictional and biaxial effects induced by the clamping force.

Table 3: Experimental table for tension/compression tests of SPCC and DP590.

\begin{tabular}{cccccc}
\hline \multirow{2}{*}{ Material } & Displacement & \multicolumn{5}{c}{ Strain rate $[/ \mathrm{sec}]$} \\
\cline { 3 - 6 } & {$[\mathrm{mm}]$} & 0.001 & 0.01 & 0.1 & 1.0 \\
\hline \hline \multirow{3}{*}{ SPCC } & 1.0 & $\mathrm{O}$ & $\mathrm{O}$ & $\mathrm{O}$ & $\mathrm{X}$ \\
& 2.0 & $\mathrm{O}$ & $\mathrm{O}$ & $\mathrm{O}$ & $\mathrm{X}$ \\
& 3.0 & $\mathrm{O}$ & $\mathrm{O}$ & $\mathrm{O}$ & $\mathrm{O}$ \\
& 4.0 & $\mathrm{O}$ & $\mathrm{O}$ & $\mathrm{O}$ & $\mathrm{O}$ \\
\hline \multirow{3}{*}{ DP590 } & 1.0 & $\mathrm{O}$ & $\mathrm{O}$ & $\mathrm{O}$ & $\mathrm{X}$ \\
& 2.0 & $\mathrm{O}$ & $\mathrm{O}$ & $\mathrm{O}$ & $\mathrm{X}$ \\
& 3.0 & $\mathrm{O}$ & $\mathrm{O}$ & $\mathrm{O}$ & $\mathrm{O}$ \\
\hline
\end{tabular}

\subsection{Correction of acquired load}

All acquired stress-strain results require corrections for frictional and biaxial effects induced by the clamping force, $F_{c}$, because the specimen is compressed by the spring-loaded clamping device in the thickness direction to suppress T-buckling. The measured force from the load cell, $F_{m}$, is the addition of the force from the specimen deformation, $F_{d}$, and the frictional force, $F_{f}$.

$$
F_{d}=F_{m}-F_{f}
$$

The friction behaviour is represented by the Coulomb friction law as follows $[1,2]$ :

$$
F_{f}=\mu F_{c}
$$

The frictional conditions can be changed according to the material, the geometrical change of a specimen and the accumulated damage to the Teflon film. The friction coefficient is generally known to be in the range of $0.03 \sim 0.09$ when the Teflon film is used for tension/compression tests [2-4]. The friction coefficients for SPCC and DP590 are selected as 0.06 and 0.08, respectively, to correct the acquired curve obtained from the tensile test with clamped into a 
corrected curve by comparison with the acquired curve obtained from a tensile test with unclamped.

In order to subtract the through-thickness stress, $\sigma_{t}$, induced by the clamping force, the von Mises yield function is utilized to calculate the effective stress corrected from the biaxial effect.

$$
\bar{\sigma}=\sqrt{\frac{1}{2}\left[\left(\sigma_{m}-\sigma_{t}\right)^{2}+\sigma_{m}^{2}+\sigma_{t}^{2}\right]}
$$

where $\sigma_{m}$ is the measured stress from the load cell. After both frictional and biaxial corrections, the flow curve of the clamped specimen agrees well with that of the unclamped specimen as shown in fig. 8.

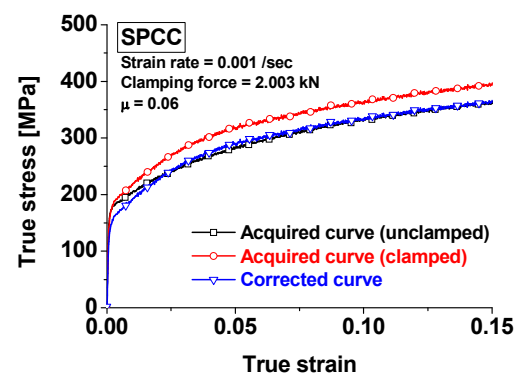

(a)

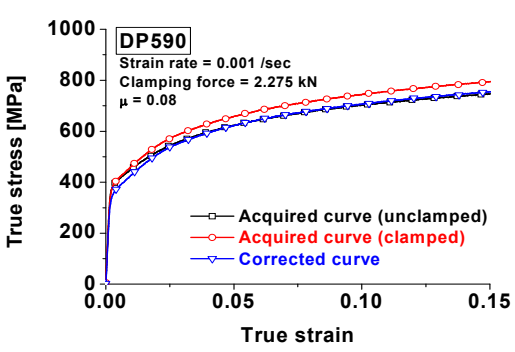

(b)

Figure 8: Frictional and biaxial corrections in the uniaxial tension state: (a) SPCC, 0.001/sec; (b) DP590, 0.001/sec.

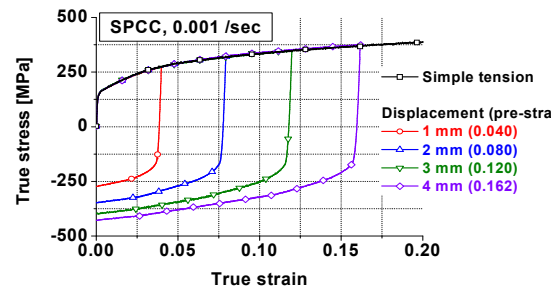

(a)

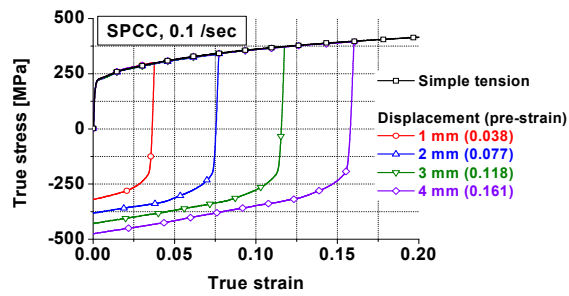

(c)

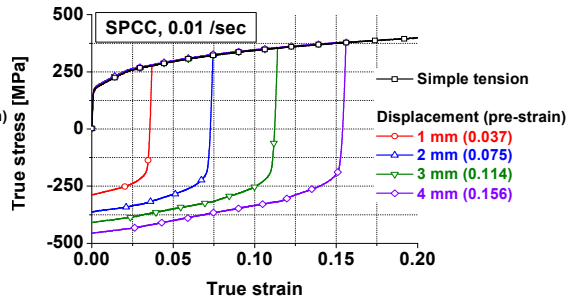

(b)

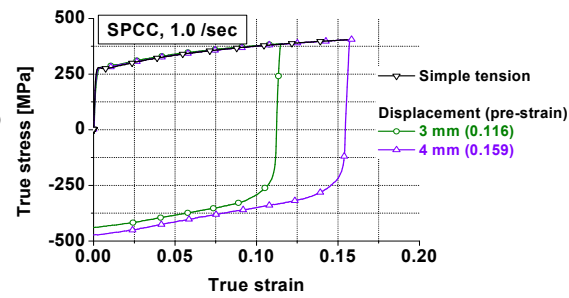

(d)

Figure 9: Tension/compression test results of SPCC at various strain rates: (a) $0.001 / \mathrm{sec}$; (b) $0.01 / \mathrm{sec}$; (c) $0.1 / \mathrm{sec}$; (d) $1.0 / \mathrm{sec}$. 


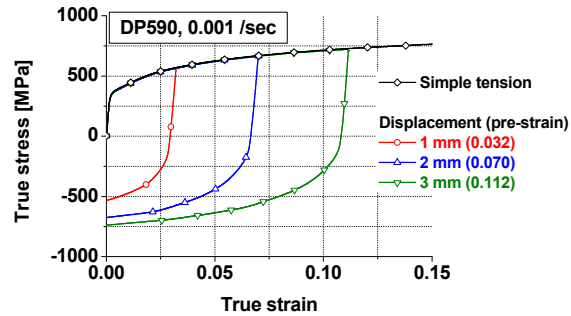

(a)

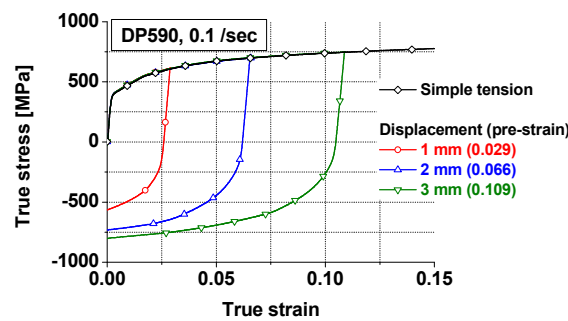

(c)

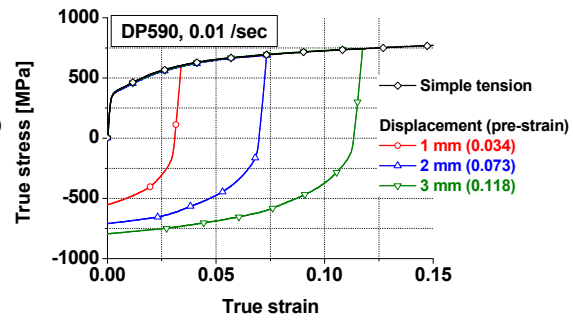

(b)

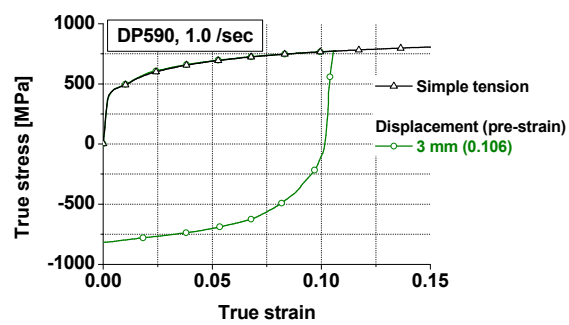

(d)

Figure 10: Tension/compression test results of DP590 at various strain rates: (a) $0.001 / \mathrm{sec}$; (b) $0.01 / \mathrm{sec}$; (c) $0.1 / \mathrm{sec}$; (d) $1.0 / \mathrm{sec}$.

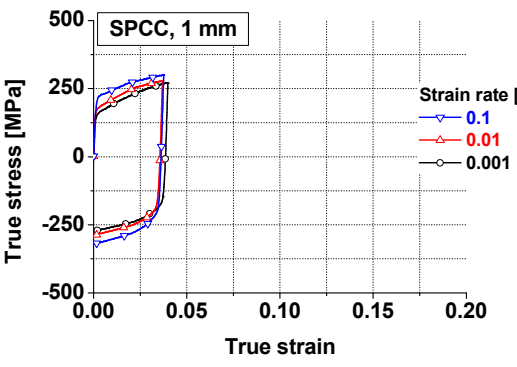

(a)

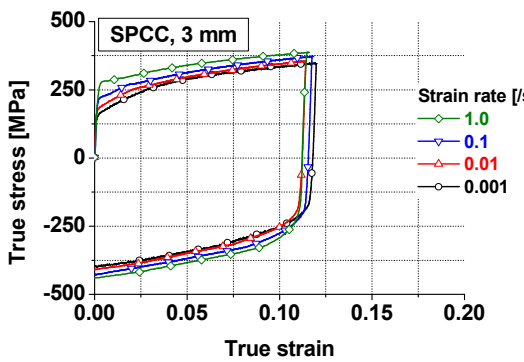

(c)

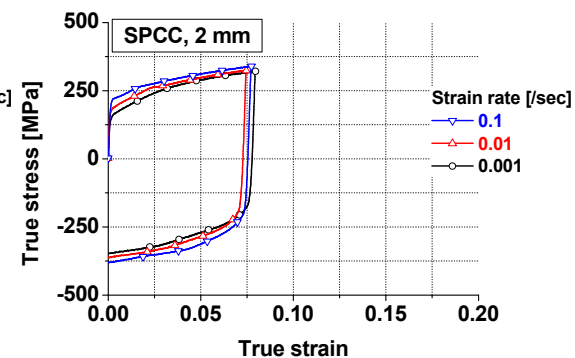

(b)

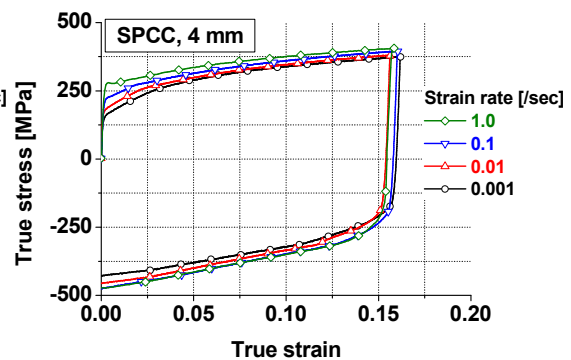

(d)

Figure 11: Tension/compression test results of SPCC at various actuator displacements (pre-strains): (a) $0.001 / \mathrm{sec}$; (b) $0.01 / \mathrm{sec}$; (c) $0.1 / \mathrm{sec}$; (d) $1.0 / \mathrm{sec}$. 


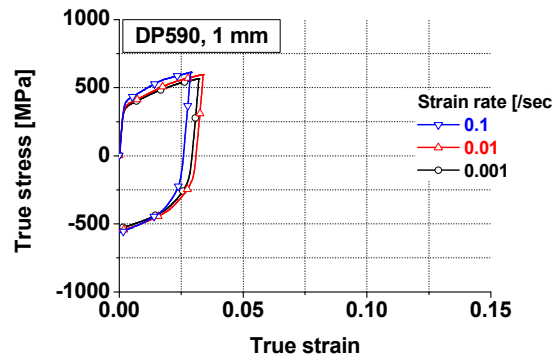

(a)

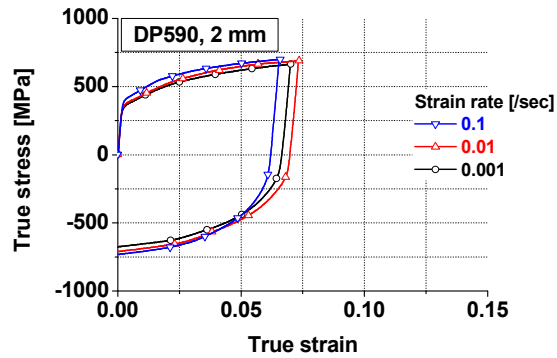

(b)

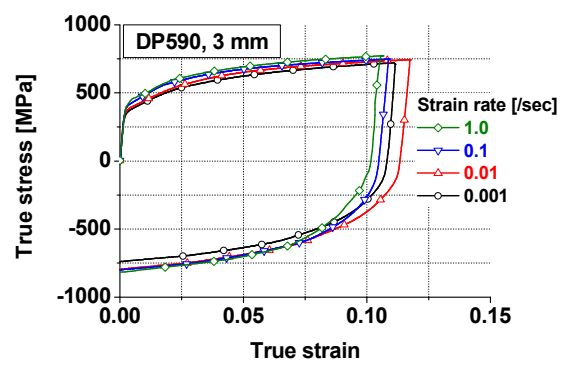

(c)

Figure 12: Tension/compression test results of DP590 at various actuator displacements (pre-strains): (a) $0.001 / \mathrm{sec}$; (b) $0.01 / \mathrm{sec}$; (c) $0.1 / \mathrm{sec}$.

\section{Hardening behaviour}

True stress-true strain curves were obtained after frictional and biaxial corrections, as shown in figs. 9 and 10. Hardening curves expand with the increase of the pre-strain at various strain rates. To investigate the strain rate effect on the hardening behaviour, true stress-true strain curves were redrawn with the variation of the strain rate as shown in figs. 11 and 12. Hardening curves also expand with the increase of the strain rate at various pre-strains. It is well known that the strain and the strain-rate hardening are caused by micro-structure changes inside the materials. To confirm this general expectation, Huh et al. [9] demonstrated by observing TEM experimental results that the mechanism of the strain-rate hardening is the change of the dislocation structures and the increase of the dislocation density. The mechanism of the hardening behaviour during tension/compression loading also can be explained by the micro-structure change of steel sheets caused by the pre-strain and the strain rate effect. From the experiments, it is clear that the tension/compression hardening behaviour is changed by the strain rate as well as by the pre-strain. This means that the prestrain and the strain rate effect on the tension/compression hardening behaviour should be considered simultaneously to improve spring-back predictability in numerical sheet metal forming simulation. 


\section{Conclusions}

This paper investigates tension/compression hardening behaviour by performing tension/compression tests using a newly developed spring-loaded clamping device. Contributions in this paper are summarized as follows:

1) A specimen shape was designed with the secant formula and the Euler method adopted by Boger et al. [2] to prevent L-buckling and W-buckling in the desired tension/compression test range. The clamping force to suppress $\mathrm{T}$ buckling was calculated with the plate buckling theory. A spring-loaded clamping device was newly developed to suppress T-buckling in the tension/compression test. From experiments, it is noted that the dimension of a specimen and the choice of compression-type coil springs are appropriate to execute reliable tension/compression tests.

2) The strain in the gauge region of a clamped specimen was measured directly by using a digital image processing technique in order to overcome the inherent structural problems in the use of conventional contact-type extensometers. The measuring scheme using the digital image processing technique can provide accurate strain history in the gauge region of a clamped specimen during tension/compression tests.

3) With the test conditions established, hardening curves were reliably obtained for various pre-strains and strain rates. The tension/compression hardening curves were expanded with the increase of the strain rate as well as with the increase of the pre-strain. The mechanism of the pre-strain and strain rate hardening was explained by micro-structure changes, such as dislocation structures and dislocation density. Experiments provide good information of the hardening behaviour with respect to the pre-strain and the strain rate.

4) The newly developed clamping device can be directly applied to tension/compression tests at high strain rates because of its compactness and convenience to control the clamping force with various tensile testing machines. This device guarantees the response of the clamping pressure in real time when the clamping condition is changed by the specimen deformation during tension/compression tests.

\section{References}

[1] Lee, M. G., Kim, D., Kim, C., Wenner, M. L., Wagoner, R. H. \& Chung, K., Spring-back evaluation of automotive sheets based on isotropic-kinematic hardening laws and non-quadratic anisotropic yield functions, part II: characterization of material properties. Int. J. Plast., 21, pp. 883-914, 2005.

[2] Boger, R. K., Wagoner, R. H., Barlat, F., Lee, M.-G. \& Chung, K., Continuous, large strain, tension-compression testing of sheet material. Int. J. Plast., 21, pp. 2319-2343, 2005.

[3] Cao, J., Lee, W., Cheng, H. S., Seniw, M., Wang, H. P. \& Chung, K., Experimental and numerical investigation of combined isotropic-kinematic hardening behavior of sheet metals. Int. J. Plast., 25, pp. 942-972, 2009. 
[4] Lee, M. G., Kim, D., Kim, C., Wenner, M. L. \& Chung, K., Spring-back evaluation of automotive sheets based on isotropic-kinematic hardening laws and non-quadratic anisotropic yield functions. part III: applications. Int. J. Plast., 21, pp. 915-953, 2005.

[5] Taherizadeh, A., Ghaei, A., Green, D. E. \& Altenhof, W. J., Finite element simulation of springback for a channel draw process with drawbead using different hardening models. Int. J. Mech. Sci., 51, pp. 314-325, 2009.

[6] Cao, J. \& Wang, X., An analytical model for plate wrinkling under tri-axial loading and its application. Int. J. Mech. Sci., 42, pp. 617-633, 2000.

[7] Huh, H., Kim, S. B., Song, J. H. \& Lim, J. H., Dynamic tensile characteristics of TRIP-type and DP-type steel sheets for an auto-body. Int. J. Mech. Sci., 50, pp. 918-931, 2008.

[8] Huh, H., Lim, J. H. \& Park, S. H., High speed tensile test of steel sheets for the stress-strain curve at the intermediate strain rate, Int. J. Automotive Technol., 10(2), pp. 195-204, 2009.

[9] Huh, H., Yoon, J. H., Park, C. G., Kang, J. S., Huh, M. Y. \& Kang, H. G, Correlation of microscopic structures to the strain rate hardening of SPCC steel. Int. J. Mech. Sci., 52, pp. 745-753, 2010. 